\title{
nota informativa sobre teorias probabilísticas de seguridad en estructuras
}

J. M. ANTON CORRALES, Ingeniero de Caminos

1. Sumeroio

El artículo, informativo, recuerda brevemente los principios, historia y desarrollo de las teorias probabilísticas para determinar coeficientes de seguridad. Recuerda que primero se concibe $y$ luego se calcula, recuerda los principios ya universales por su sencillez $y$ evidencia. Cita brevemente algunos nombres que contribuyeron a la teoría. El autor trabajó en ella en el Instituto Eduardo Torroja para el C.E.C.M. (Convención Europea de la Construcción Metálica), bajo la dirección de J. Batanero.

\section{Introduceión al probleme}

A) Idea de optimización

Todo quehacer lleva consigo un cierto esfuerzo; si es pequeño, se aumenta el riesgo de que salga mal; si excesivo, se pierden energías, tiempo o dinero sin compensación de ventajas significativas. Esto ocurre al cruzar la calle (se toma precaución), al dinamitar un puente (se gasta la suficiente trilita), o al construir una casa. Es el problema de la optimización, que afortunadamente suele resolverse a ojo sin cálculos. Si no, todos deberíamos llevar una calculadora en el bolsillo.

Sin embargo, hay casos concretos e importantes en que calcular mejora el conocimiento hasta el punto de ser imprescindible o normativo. Así ocurre hoy en día con las comprobaciones de resistencia en construcción.

En estas comprobaciones se estiman las cargas que van a actuar, se estima su valor elevado improbable, y en combinaciones desfavorables de diversas cargas, se reparten esfuerzos que se comparan con esfuerzos resistentes de elementos calculados con resistencias de materiales. En estos cálculos se mayoran cargas y se aminoran resistencias con unos coeficientes de seguridad. Se hace una optimización, en cuanto que se limita el material al mínimo que da seguridad cercana a la absoluta.

\section{B) Primero concebir, luego comprobar}

Es obvio que lo primero que hay que hacer es concebir bien lo que se construye, y evitar todos los errores que no detectará el cálculo. Se logra, además de la seguridad, el que la construcción sea estética y sirva para lo que se proyecta. Para ello hacen falta experiencia técnica o arquitectónica y se usan cálculos aproximados de dimensionamiento.

En general, las averías o catástrofes ocurren por errores de concepción o por una ejecución francamente mala. Hay una serie de reglas de buena práctica (no poner pórticos separados en las casas, no tener mecanismos, el yeso no atacará la estructura, no cruzar tres cordones de soldadura, no poner la casa en el cauce de inundaciones de un río...) fundamentales que no son representables por coeficientes de seguridad. En el fortísimo terremoto de Agadir se cayeron principalmente casas mal construidas... Y es raro que estas averías se produzcan por ser los esfuerzos de las cargas superiores a los esfuerzos resistentes.

Sin embargo, el poder garantizar que los esfuerzos debidos a las cargas no sobrepasan los esfuerzos resistentes, y con un mínimo de material, da seguridad y economía. Inmediatamente se ve que con coeficientes de seguridad altos se gasta demasiado y con bajos el riesgo de ruina es grande. Por lo tanto, debe haber un óptimo, por lo menos teórico, en que se minimiza un coste de construcción, más un coste «temido" de ruina, en general producto de un coste de ruina por la probabilidad de esta ruina. Necesitaremos, pues, dar nociones probabilistas.

Como lo único que garantizan los coeficientes de seguridad es que los esfuerzos debidos a las cargas no sobrepasan los esfuerzos debidos a las resistencias, no podemos usar más que los riesgos de ruina debidos a esta superación de resistencias por las cargas para determinar coeficientes. 


\section{C) El problema conduce a un enfoque probabilístico}

Que todo lo que el hombre hace se puede caer es obvio. Además, puede hacerse una idea de ello considerando distribuciones estadísticas.

Los factores aleatorios que entran en juego son:

- El conocimiento a priori de las cargas.

- De las resistencias.

- Hipótesis varias de reparto de cargas, cálculo, errores de cálculo.

- Probabilidades de coincidencia de máximos de diversas cargas.

Para cuantificar estos hechos se hacen hipótesis matemáticas en cascada, cada vez más precisas pero menos evidentes. Al final, llegan a modelos matemáticos muy personales y siempre algo discutibles.

\section{D) Definiciones y principios bien establecidos y confrontados}

1. Cargas:

En primer lugar hace falta dar una definición de carga que será la característica, basada en leyes probabilistas. Como se puede definir de muchos modos, se da una definición tal que sea equiparable la carga característica a las cargas "máximas», "excepcionales", o de las normas, de modo que cargas de definición probabilista vayan reemplazando progresivamente las cargas pretendidas máximas. Ello es necesario, pues son organismos distintos los que fijan cargas y los que dan normas para materiales, y no van a cambiar sus normas a la vez.

Al fijar cargas entre usuarios conviene se den unas cargas características (caso de la obra civil de una instalación industrial). Es necesario que las cargas características para hormigón sean las mismas que para acero, o sea, es necesaria una definición común de carga característica.

Los principios de definición de cargas que parecen estar adoptados y no contener errores o declaraciones que vayan a quedarse fuera de posteriores teorías, están contenidos en nuestra siguiente declaración (interna para el C.E.C.M.):

Habiendo algunas discrepancias sobre las definiciones fundamentales a utilizar en las normas, exponemos nuestra postura, de acuerdo con las directrices establecidas en el Instituto Eduardo Torroja y adoptadas en normas internacionales (C.E.B.) como base para una consideración probabilística del problema de la seguridad, por considerar que esta postura es el enfoque real del problema de la seguridad en construcción.

«Se entiende por carga nominal, $F_{n}$, aquella para la cual se propone la utilización de la estructura.

Se entiende por carga característica, $F_{k}$, la máxima previsible, no excepcional, durante la vida de la estructura (Instrucción H.A. 61 para hormigón del Instituto Eduardo Torroja. 3.6.4).»

No se conocen, en general, los valores que va a tomar una carga $F$, pero sí se tiene una idea sobre su distribución estadística antes de concebir o comprobar la estructura. Por ello, se define carga característica como una carga que tiene una probabilidad a priori dada de ser sobrepasada durante la vida de la estructura (1). Si se supone que el tipo de distribución, o sea la distribución de la variable

(1) Esta definición de probabilidad a priori es rigurosa, y a la vez es la que mejor se intuye. Tiene en cuenta el hecho de que tanto la carga como el conocimiento de su ley de distribución son aleatorias, dando lugar a una ley combinada de distribución a priori; la característica será menor cuanto mejor se estime la ley de cargas, e igual probabilidad de sobrepasarla. Estudiar la historia de estructuras análogas es un medio de estimar las leyes de carga.

La "vida previsible" es una noción necesaria de algún modo. Quizás conviniese tomarla como magnitud aleatoria, pues lo es ciertamente. Lo cierto es que limitar ( $y$, por consiguiente, hacer limitar a los que hagan las normas nacionales) los estudios a la consideración de probabilidades de ruina anuales es insuficiente, sobre todo si se comete el error de decir que "la probabilidad de ruina en 50 años es 50 veces la probabilidad de ruina en un año", pues las probabilidades de ruina en cada año no son sucesos "independientes y exclusivos"; la probabilidad de ruina en 50 años es mucho menor que 50 veces la probabilidad de ruina en un año para el mismo edificio con las mismas exposiciones a cargas cada año.

Las funciones de distribución de cargas permanentes pueden ser constantes con el tiempo, pero no las de viento o nieve cuyas medias aumentan con el tiempo; por ello los resultados válidos para un año se distorsionan con los años. Para dar una primera idea de estos fenómenos se pueden usar leyes de valores extremos. Uno de los resultados a que conducen estas leyes es que el factor que influye en el aumento de cargas con el tiempo es la dispersión de las cargas, pero no la dispersión del conocimiento de las cargas. 
estandarizada, es conocido, de media $F_{m}$ y dispersión $\delta$, dar la probabilidad significa definir un $K$ tal que $F_{k}=F_{m}(1+K \delta)$ y viceversa.

«Carga nominal es aquella para la cual se propone la utilización de la estructura.»

«La fijación de los valores nominales y característicos de las cargas corresponde, en general, a los Organismos competentes y, en casos muy particulares, al propio proyectista.» Según la Instrucción E.M. 62, 4.2, del Instituto Eduardo Torroja (1).

No se confundirán los conceptos de carga característica y nominal.

«4.5... Las sobrecargas de explotación previsibles se tomarán de los reglamentos o normas oficiales que puedan estar en vigor para los diferentes grupos de construcciones. Salvo calificación expresa de las mismas por los Organismos competentes, se considerarán aquellas como sobrecargas características...». Instrucción E.M. 62 del Instituto Eduardo Torroja.

«4.5... Si no están definidas dichas sobrecargas de explotación en forma oficial, el proyectista fijará los valores característicos que haya de utilizar en sus cálculos, de acuerdo con lo indicado en 4.22.» (El artículo 4.22 es la definición de característica). E.M. 62, obra citada.

«4.51... En los inmuebles de varias plantas, destinados a viviendas o a oficinas que no sean de acceso público general, se considerarán, para el cálculo de soportes, muros resistentes, macizos de cimentación o cualquier otro elemento que haya de recibir las cargas de varias plantas, las reducciones, en la suma de las sobrecargas características de explotación, que autoricen las reglamentaciones vigentes.» E.M. 62, obra citada.

I. Para los comentarios del artículo 1.512 de la propuesta de redacción ISO/TC/98, del Dr. Rowe, proponemos se den las indicaciones de las definiciones de ley de distribución de cargas, carga nominal y característica, y del hecho que ambas se tomarán de las normas por el proyectista, o se fijarán por éste en defecto de norma de acuerdo con las definiciones anteriores. Deben evitarse principios inútiles o falsos que coarten las investigaciones futuras, como el de que «las leyes de carga son normales" o de que "hay que confundir carga nominal y característica» (pues hacen falta dos nociones distintas) o de que "sólo existen probabilidades de ruina anuales».

II. Creemos que las normas deben estar dirigidas a la práctica, pero fundadas en las nociones probabilistas. De acuerdo con Mr. Loof y Mr. Godfrey, afirmamos no se deben perder las nociones y definiciones apropiadas que a través de Organismos nacionales (Instituto Eduardo Torroja)... e internacionales (C.E.B., propuesta por I.S.O., propuesta C.E.C.M.) son ya de dominio general, sin perjuicio de enriquecimientos por nuevos trabajos una vez depurados.

O sea, que se calculará con las cargas de las normas y se pondrá, cuando proceda, en las obras una placa, con la carga nominal igual a $1 / K^{\prime}$ veces la característica, con $K^{\prime}$ fijado por las normas. En el futuro las normas, en vez de fijar cargas «excepcionales», fijarán cargas con una probabilidad dada de ser sobrepasadas, por ejemplo el $5 \%$ (Comité Europeo del Hormigón), que se asimilan bien a las anteriores. La I.S.O. piensa poner $K^{\prime}=1$, es decir, confundir carga nominal y características.

Para cargas estabilizantes hay menos acuerdo. Se suelen considerar sin minorar. A veces se minoran.

La carga que delimita responsabilidades entre usuario y proyectista es la nominal y, en su defecto. la característica. De hecho siempre es en parte la característica.

\section{Resistencias:}

Hay discrepancias sobre las definiciones de cargas a emplear. No las hay sobre las resistencias. Suele tomarse una resistencia característica que tenga una probabilidad pequeña de ser sobrepasada. Se suele admitir que las resistencias siguen una ley normal. Si la media es $O_{m}$ y la dispersión $\delta$ se suele tomar $(1+1,64 \delta) Q_{m}$ en hormigón ó $(1+2 \delta) Q_{m}$ en construcción metálica, correspondiendo a 5 y $2,7 \%$ de probabilidades respectivas de tener resistencias menores.

(1) En particular, para una carga nominal propuesta por el usuario puede el Organismo competente fijar cuál es la característica conveniente para que, a la vista de la nominal, el usuario se comporte de modo que se respete la definición de característica.

Ciertas normas españolas (del Instituto Eduardo Torroja en particular) proponen en el caso general que la carga característica sea $K^{\prime}$ veces la nominal con $K^{\prime}=1,1$. Creemos que en casos particulares proceden otros valores de $K_{0}^{\prime}$. 
De paso se comprueba que, dentro de ciertos límites, a igual caracteristica, igual probabilidad de ruina. Son equivalentes, pues, dos materiales de igual característica, uno con mayor media y dispersión que el otro.

Recordemos (Dr. Ferry Borges: O Dimensonamento de Estructuras, Laboratorio Nacional de Engenharia Civil) que esta resistencia sólo está conocida para un "elemento resistente» dado. Varía para un mismo material con la forma (probetas de hormigón), tipo (viga o cordón de soldadura). En particular decrece si se unen frágilmente elementos; la unión es frágil si al romperse uno sólo se rompe todo. Dúctil si tienen que romperse todos para que se caiga. Ejemplo de lo primero son cables empalmados; de lo segundo, cables en paralelo o rótulas en cálculo plástico. Caben intermedios para los que las teorías se complican.

\section{Otros:}

Las incertidumbres de cálculo son todavía más vidriosas que las de cargas. En ellas están las de cálculo de elementos compuestos, cálculo de estructuras, errores de cálculo y simplificaciones varias. La probabilidad de ruina depende mucho de estas incertidumbres.

\section{A qué se aplican los coeficientes:}

Las leyes de cálculo pueden no ser lineales. Recordemos que los desplazamientos debidos a las cargas suelen ser más que proporcionales a las cargas, y pueden aumentar los esfuerzos debidos a éstas; estos esfuerzos aumentar los desplazamientos y así sucesivamente.

Véase un ejemplo de lo que pasa al mayorar cargas o minorar resistencias por un coeficiente 2, cuando la carga es 1 , y el esfuerzo es el cuadrado de la carga. Si se mayoran cargas, hace falta una resistencia de 4. Si se minoran resistencias, basta con 2 .

Parece más real y más seguro, en los casos de pandeo, que son los más comprometidos, el mayorar las cargas que el minorar resistencias. Se puede dividir el coeficiente de seguridad en dos factores: uno mayorando cargas, otro minorando resistencias. Se justifica esto diciendo que en caso de ruina influye tanto el exceso de cargas como la falta de resistencias. Este razonamiento intuitivo parece intuitivamente bueno, siempre que se mayoren más las cargas, que minoren las resistencias. Es cómodo para el hormigón armado, pues se minora más su resistencia (por 1,6 en H.A. 57) que la del acero (1,2 en H.A. 57). Tal hizo la norma H.A. 57 del Instituto Eduardo Torroja y tal hicieron muchas otras normas nacionales e internacionales de hormigón armado y pretensado. En los casos de pandeo se emplearon varios sistemas para linealizar el problema (método Omega para acero de la excentricidad mayor para hormigón), siendo mejor mayorar sólo cargas y no minorar resistencias.

\section{Costo de la ruina:}

La gravedad de las consecuencias de la ruina, la duración del edificio, la disponibilidad de capitales que permita construir muy seguro u obligue a construir barato y el hecho de aumentar las cargas con el del peso propio en mayor o menor cuantía, son muy importantes para fijar coeficientes de seguridad, sobre todo el primero.

\section{Coincidencia de cargas:}

Uno de los puntos más delicados es el saber si diversas cargas coinciden por ser debidas a una misma causa, o si sus máximos tienen poca probabilidad de coincidir. Se reduce la carga de cálculo en un edificio para pisos cuando se cargan todos los pisos, porque no suele haber un entierro en todos los apartamentos a la vez, pero no se reduce al considerar los pisos de un teatro o cine, pues puede que se llenen todos, por ejemplo por poner una obra buena. El estudio de estas coincidencias es difícil y no está hecho - hemos hecho un modelo matemático que vale, si se toman precauciones-, y es fundamental para reducir coeficientes de seguridad de modo responsable.

\section{7.' Cargas excepcionales:}

Se pueden distinguir cargas «excepcionales», que se presentan poco, para las que se admiten averías, cuya repetición sería más peligrosa; y «usuales», cuya repetición es corriente. Como toda carga característica es grande conviene reservar el nombre de «excepcional» a cargas de probabilidad menor de $1 / 10$ de ocurrir en un año, por ejemplo. Para ellas se pueden reducir coeficientes, admitir daños ligeros y considerar importantes reducciones en otras cargas al sumar. Piénsese en cargas sísmicas. 


\section{E) Tipos de comprobación}

Una vez convenientemente mayoradas cargas y minoradas resistencias en tipos de cargas, se procede a:

\section{Cálculos aproximados:}

No tienen validez legal, pero se hacen para obras pequeñas, o partes pequeñas de obras grandes, siendo muy útiles para dimensionar.

2. Cálculos en rotura pura que dicen si se cae o no la estructura:

En general, cuando dicen que se cae, ha habido muchas plastificaciones o similares poco reversibles. Dicen mucho de la reserva de seguridad, y son fuente de investigación, pues se conocen mal. El pandeo plástico está mal estudiado y requiere una fina intuición al estudiar cada caso. La rotura depende del orden de aplicación de cargas a menudo. Todo esto hace que no se use, pero tenga porvenir el cálculo plástico de estructuras complejas.

\section{3.- Reparto elástico, comprobación en "rotura" o en fin de deformaciones reversibles de elementos:}

Es el que tiende a extenderse. El reparto elástico se conoce bien. Para acero se considera rotura el sobrepasar el límite elástico en alguna parte. En hormigón se hace un cálculo de rotura de sección (por rotura de hormigón y límite elástico de aceros).

No se suelen tener en cuenta reservas de plasticidad hiperestáticas.

Se toman menos garantías a deformación que a rotura.

Los casos normales de pandeo se resuelven mediante reducciones o cargas normales (método omega y otros). Se llega en pandeo de vigas a determinar leyes de resistencias experimentales para secciones y longitudes dadas (trabajos del C.E.C.M., bajo la dirección de Sfintesco), hallando medias y dispersiones de resistencias. Son mejores que las teorías matemáticas tipo Euler y derivadas (pandeo lateral; pandeo de alma). Estas últimas suelen ser pesimistas y van muy bien en los casos, muy numerosos, en que no hay experiencias. El considerar deformaciones secundarias ya es un estudio de pandeo.

En general, al llegar a lo que se toma por rotura y que suele ser fin de deformaciones reversibles, siguen valiendo las leyes de reparto elástico. Se tiene reserva de seguridad a la ruptura final.

\section{Reparto elástico, cálculo elástico:}

Difiere del anterior por prioridad histórica:

Los criterios de fin de deformaciones reversibles son más antiguos. Su defecto es que consisten en minorar resistencias. Siempre que hay deformaciones secundarias, están del lado de la inseguridad.

Recordemos que se estudian "deformaciones secundarias» cuando se tiene en cuenta la variación de esfuerzos debida a los desplazamientos originados por las cargas. El límite es el pandeo.

\section{F) Coeficientes propuestos}

Aunque un caso de carga, $S_{i}$, no es un número, sino un "vector» conjunto de fuerzas sobre una estructura, se puede representar una reunión de casos, $S_{i}$, con intensidades multiplicadas por $C_{i}$ en forma $\Sigma C_{i} S_{i}$. $\mathrm{Si}$ el reparto es lineal, se convierte la suma vectorial en sumas aritméticas.

Hay que tener en cuenta que diversas cargas necesitan diversos coeficientes. $Y$ que al intervenir cargas de naturaleza distinta, sus máximos no coinciden, y si lo hiciesen darían una ley aleatoria cuya característica sería inferior a la suma de las características. El medio de representación a la vez potente y sencillo es de los coeficientes de ponderación. Se introducen en expresiones $\sum_{i} S_{i} C_{i} e_{i l}$; la estructura resis-

tirá a todas las sumas $l$ de una familia. El sólido $\left(S_{i}>0\right.$ para todo $i, \Sigma S_{i} C_{i} e_{i l}<1$ parte todo $\left.l\right)$ representa bien una familia de coeficientes de ponderación.

Si hay dos cargas, se pueden representar en el plano (figura 1.1). Si

$$
\begin{aligned}
& S_{n}=\text { nieve = carga }(1), \\
& S_{v}=\text { viento = carga }(2), \\
& S_{t}=\text { temperatura, } \\
& S_{p}=\text { permanente, } \\
& S_{u}=\text { uso, }
\end{aligned}
$$


una presentación puede ser:

"tómese la resistencia mayor de

$$
\begin{aligned}
& 1,33\left(S_{p}+S_{t}\right)+S_{u}(1,5), \\
& 1,33\left(S_{p}+S_{t}\right)+S_{p}(1,5), \\
& 1,33\left(S_{p}+S_{t}\right)+S_{v}(1,5), \\
& 1,33\left(S_{p}+S_{t}\right)+1,4\left(S_{u}+S_{n}\right), \\
& 1,33\left(S_{p}+S_{t}\right)+1,4\left(S_{u}+S_{v}\right), \\
& 1,33\left(S_{p}+S_{t}\right)+1,4\left(S_{v}+S_{n}\right), \\
& 1,33\left(S_{p}+S_{t}+S_{v}+S_{n}+S_{u}\right) .
\end{aligned}
$$

Los coeficientes de ponderación son: 1,33; 1,5; 1,4. La figura 1.2 indica una curva teórica (equiprobabilidad de ruina).

La figura 1.3 indica una presentación de otro tipo.

Corresponde a poner las cargas en cada orden posible, mayoradas por su coeficiente de seguridad para carga aislada cada una. Se suman ponderándolas sucesivamente por números menores de $1(1,0,90,8 \ldots$, etcétera). En la figura 1.3 representamos: $1,45\left(S_{1}+\right.$ $\left.+0,9 S_{2}\right)$ y $1,45\left(S_{2}+0,9 S_{1}\right)$

Nos parece que esta representación es muy real y potente.

Dos familias que den sólidos muy parecidos son prácticamente equivalentes. Tal ocurre con las tres curvas de las figuras 1.1, 1.2 y 1.3, aunque las familias de coeficientes sean distintas en 1.1 y 1.2 .

\section{Historia sucinta de las ideas probabilísticas}

Hasta 1953 puede verse en la obra del Dr. Ferry Borges: O Dimensonamento de estructuras. Da una lista de autores:

- Max Mayer, 1926, primera tentativa.

- III Congreso de la Asociación Internacional de Puentes y Estructuras (Lieja, 1948).

- Moe (1848). Da dos coeficientes, uno minorador de resistencias medias, otro mayorador de cargas medias.

- Korangi (1948). Diferencia influencia, peso propio y sobrecarga. tema.

- Dutheil (1948) - Wästlund. Discusiones sobre el

- Kjellman (1940). Uso de probabilidades y de leyes estadísticas.

- Vasco Costa (1948). Uso de probabilidades de ruina en cálculo. Criterios económicos. Parecidos a Bret y Levi (1948); Weerzbichy (1939).

- Freudenthal, que todavía trabaja en la materia, en 1945, estudia cargas y ejemplos.

- Johnson (1953), Borges (1952).

- En 1951-52-53, Torroja y Páez publican un método completo de determinación de coeficientes de seguridad, con ábacos.

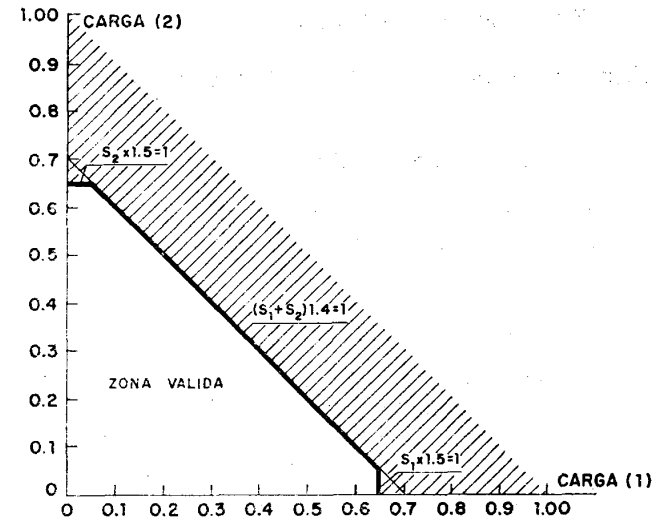

Fig. 1.1

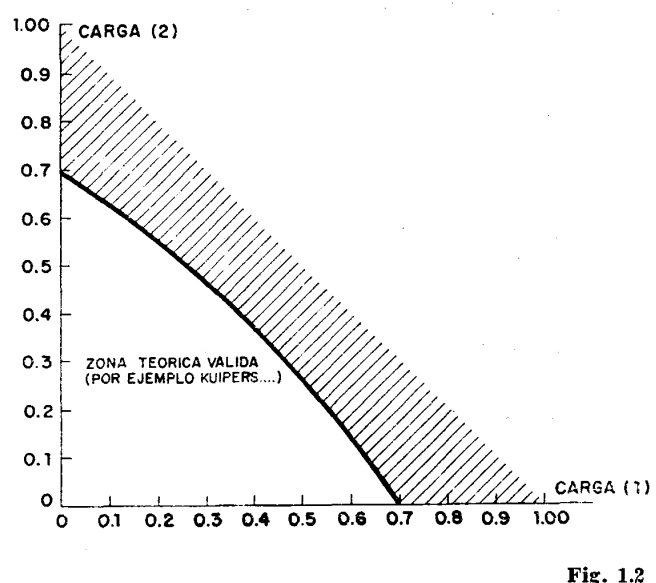

Fig. 1.2

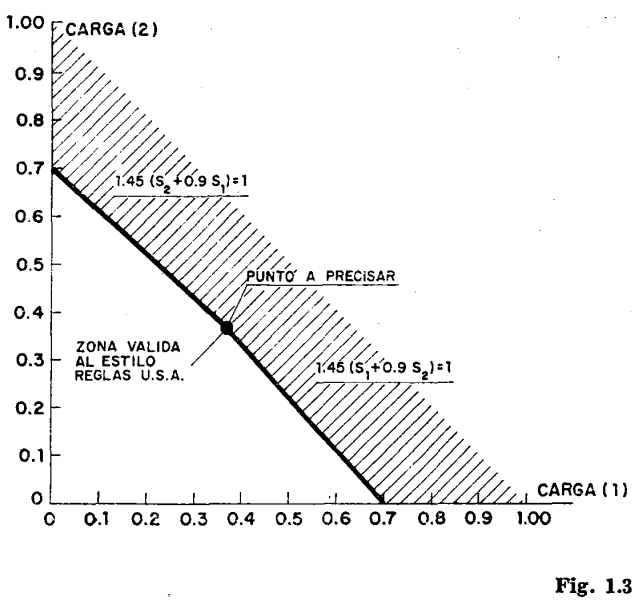


Como elemento auxiliar de cálculos usaron métodos gráficos y calculadoras mecánicas del Gabinete de Cálculo del Instituto Eduardo Torroja (hoy de Cálculo Electrónico). Son más pesados con la gran ventaja de usar curvas estadísticas reales. Sus ventajas son aminoradas por la imprecisión de conocimientos de los extremos de las curvas de probabilidad, que son fundamentales.

Consideran las distribuciones de:

$x=$ solicitación $\mathrm{real} /$ prevista;

$y=$ tensión real/tensión teórica según base teórica del cálculo;

$z=$ resultado real en cargas/resultado numérico;

$t=$ resistencia teórica/resistencia disminuida por defectos de ejecución;

$u=$ resistencia de materiales teórica/resistencia real.

Si el producto $x \cdot y \cdot z \cdot t \cdot u$ es mayor que el coeficiente $C$ de proyecto, hay ruina.

El $C$ óptimo minimiza (costo de ruina $\times$ probabilidad a priori de ruina durante la vida prevista + costo estructura). Se hallan ábacos en función de tipo de estructura (edificios, viviendas, puentes), consecuencias ruina, tipo carga (sobrecarga-peso propio), tipo de vigilancia en obra (sobre todo para hormigón).

Las bases estadísticas son relativamente pequeñas. Es el primer trabajo que da resultados concretos, lo que, unido al éxito con que fue seguido por las normas, le da gran valor. El enfoque en forma de producto sigue siendo usado hoy.

La propia obra de Ferry Borges, que Mr. Cornell, Assistant Professor en el M.I.T. (Cambridge, Massachussets, U.S.A.), habiéndola leído en portugués, calificó de «antigua de fecha, moderna de concepto", es síntesis de lo que se hizo en la época. Muchas cosas no se han mejorado, y contiene partes originales cuidando de no poner errores. Posteriormente, el autor ha trabajado en las medidas reales de cargas y resistencias en el Laboratorio de Ingeniería Civil de Lisboa, uno de los mejores en equipo y en personal del mundo.

Habla de conocimiento cierto (determinista), aleatorio (con leyes de probabilidades conocidas de cargas, reparto y resistencia), estratégico (las cargas dependen del coeficiente de seguridad, pues si es mayor, los usuarios cargarán más la estructura) e incierto (no se conocen leyes). La realidad, nos parece, es mezcla de los cuatro tipos de conocimiento, pudiendo estimarse un grado de incertidumbre. La terminología de Ferry Borges corresponde a una realidad; hemos encontrado que estas distinciones son muy relevantes. Piénsese en la carga nominal que hay que poner en un puente o en un forjado para que los usuarios se comporten de modo que haya una probabilidad pequeña de sobrepasar la carga característica (estratégico), o en la distinción entre incertidumbres debidas a las leyes aleatorias del viento (aleatorio) y debidas al conocimiento de las leyes (incierto). Son fundamentales estas distinciones, para hacer modelos matemáticos y hacer planes de medidas.

Conocida la ruina de un elemento, a partir de ella se puede hallar la de varios elementos agrupados. En particular si es grupo frágil, o sea, cuando basta que se rompa un elemento (como los eslabones de una cadena) para que se falle el conjunto, o dúctil cuando tienen que romperse todos (los alambres de un cable). Estudia casos mixtos y considera la semejanza de modelos —que interesa al Instituto de Lisboa para sus ensayos-.

Aunque se conserven semejanzas de cargas, dimensiones, deformaciones y mọ́dulos, para corresponderse la probabilidad de rotura del modelo con la de la estructura tienen que guardar cierta semejanza, las dispersiones de las resistencias de los materiales. Para un mismo material, su resistencia disminuye al tener elementos más pequeños en casos frágiles (tubos a presión: los más pequeños se calculan a veces con resistencia menor por tener menos espesor e influir más las imperfecciones).

Las leyes de semejanza se extienden al estudio aleatorio de las deformaciones.

El autor considera cargas de gravedad, viento y seísmos, haciendo síntesis de los conocimientos de entonces, en la vía de los estudios posteriores.

Estudia, con numerosas curvas, las propiedades mecánicas de acero y hormigón.

Estudia casos concretos (postes eléctricos, discriminación entre dos ofertas).

Posteriormente, el autor escribió monografías sobre teorías estadísticas de similitud estructural (Lisboa, 1961) («es la teoría de semejanza a la deformación, la más interesante. Podrá ser fácilmente generalizada...»). 
Comportamiento estructural y critécios de seguâdad (1964) donde estudia comportamiento no lineal (mé todo de Cross adaptado en ordenadas), comportamiento aléatorio no lineale(las leyés són aleatorias), y cargas dinámicas (1968), donde e estúdia el viento y los seísmos; en particular en cuanto a resonancias y ensayo dê modelos.

En general, se va a una ley de cargas (función de densidad $f(Q), Q=$ esfuerzo proporcional a lascarga: sobre un elemento) y de' resistencias [ $g(R)=$ función de densidad de la resistencia $R$ correspondiente sobre un elemento dado]: Y se hạlla la probabilidad de ruina del elemento:

$$
f(Q) g(R) d Q d R \text {, (fig } 2)
$$

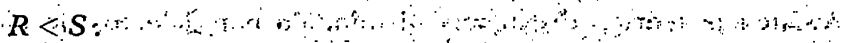

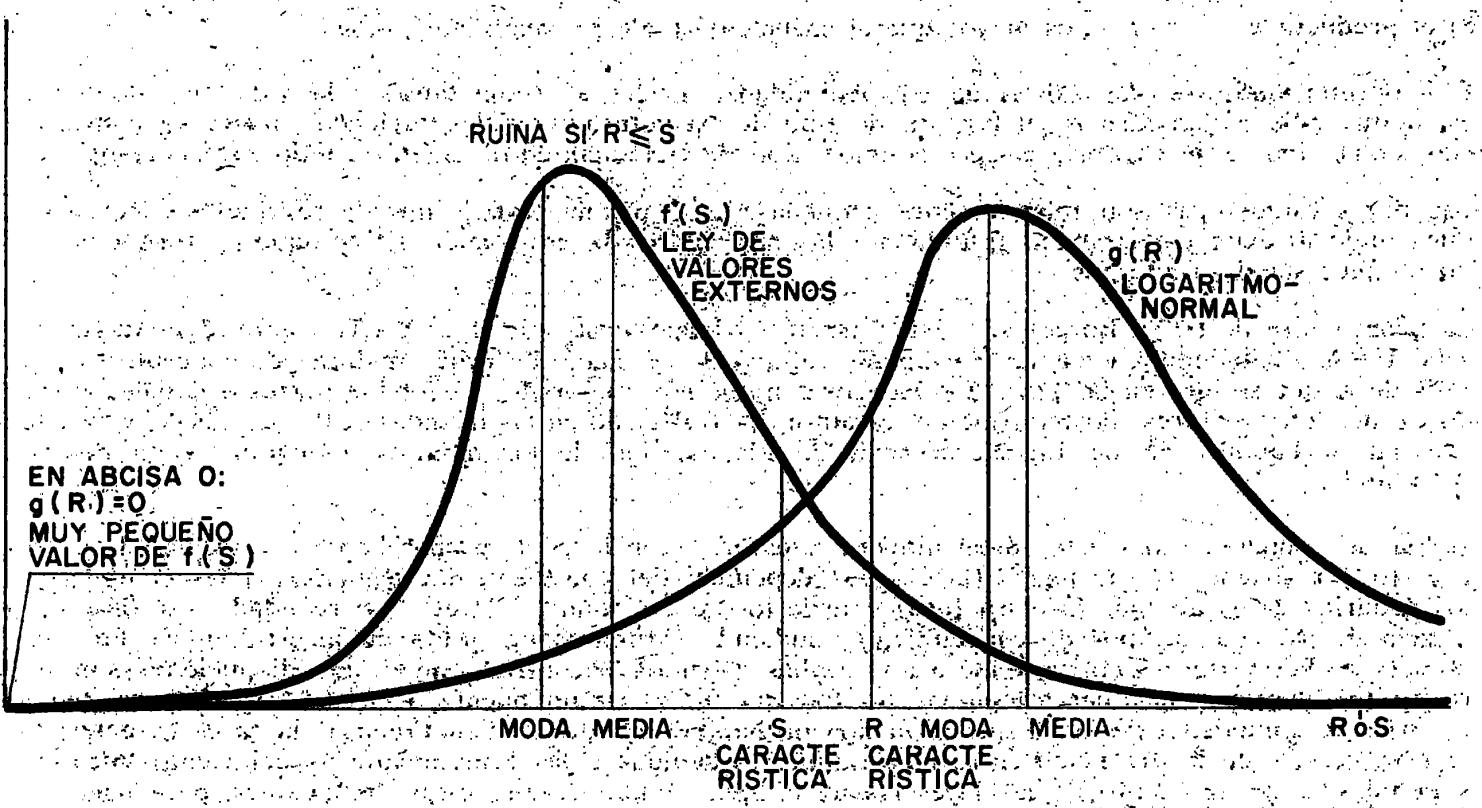

Fig: 2.1. Dispersiones exageradas

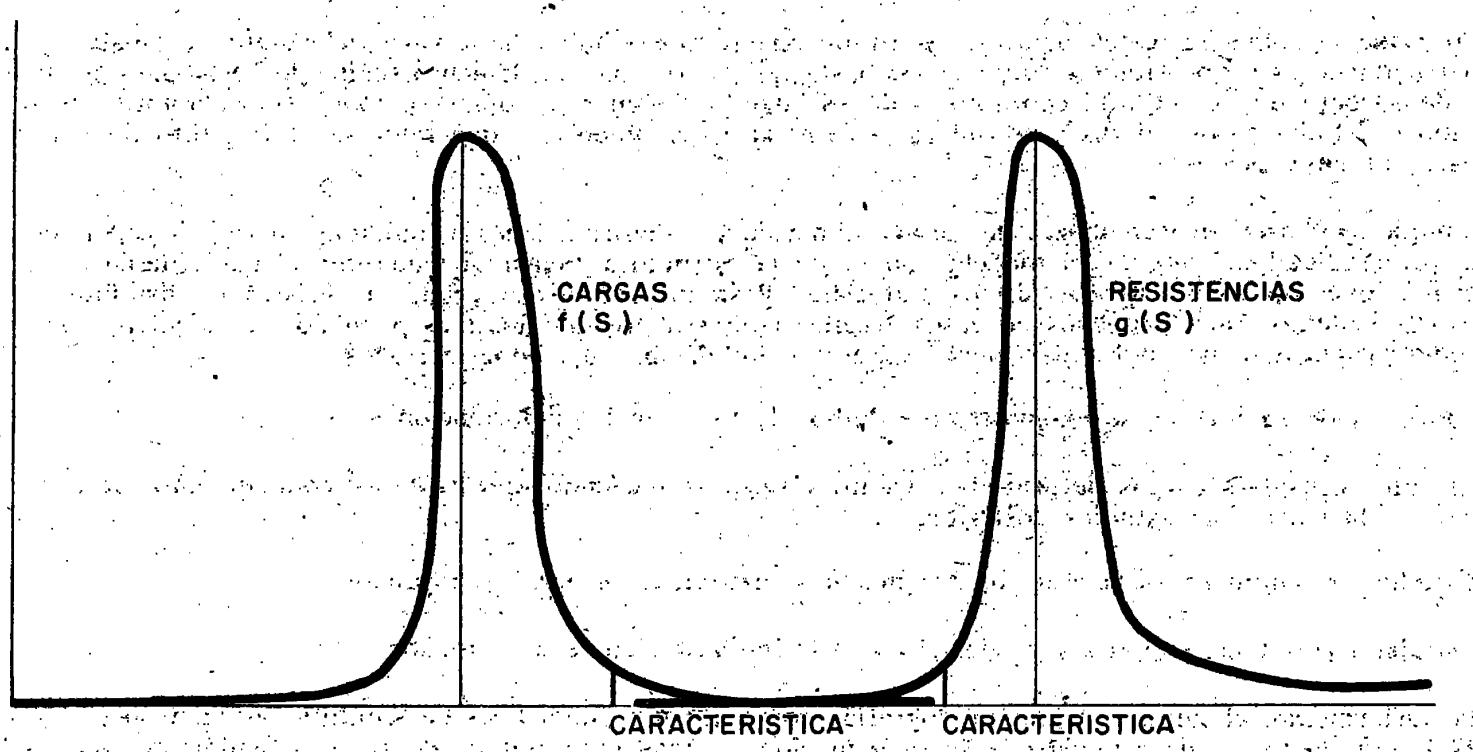

Fig. 2.2. Dispersiones realẹ 
A veces se consideran también cargas y resistencias negativas.

Las normas del Torroja (H.A.61) y (E.M. 62) dan definiciones adaptadas (característica = media más o menos una varianza) a las teorías probabilísticas. En los comentarios de H.A. 61 viene un resumen concreto y utilizable de las teorías de Páez. Se nota en estas normas una diferencia tradicional de enfoque entre hormigón y acero. Por ejemplo, para el pandeo consideran en el acero el método Omega y una cxcentricidad adicional en el hormigón (probablemente idea de Páez); se produce una deformación en rotura que da una excentricidad mayor. Se comprueba si con esa excentricidad hay o no rotura. El método es válido, salvo que existan otros experimentales mejores. Estas normas tienen varios autores (J. Batanero, J. A. Torroja, A. Páez, A. García Meseguer, C. Benito, M. Bouso, F. Cassinello, G. Echegaray, J. Nadal, J. M. Pedregal, R. Piñeiro, F. del Pozo, A. Prado, S. Rocci, J. M. Urcelay, A. Blasco Vilatela, C. Fernández Casado, R. M. Guitart, R. de Heredia, V. Mas, M. de Miró, F. Rodríguez-Avial, R. Rodríguez Borlado, M. del Campo, P. García de Paredes, C. de la Peña, F. Arredondo, J. Calleja, J. M. Flórez de Losada, D. Gaspar, J. Laorden, L. Riesgo, J. M. Tobío).

Con posterioridad se han publicado muchas notas informativas y muchos pequeños trabajos que aportan algo. Además se han empezado bastantes investigaciones que suelen comprender:

1. $\quad$ Exposición de ideas, las generalmente admitidas y alguna nueva.

2. Enfoque matemático peculiar del trabajo.

3. Modelo matemático. Estos modelos son pesados de concepción y manejo, requieren discípulos y computador (en el de Torroja-Páez, no había todavía computador y usaron métodos gráficos). Por ello son muy limitados, concretos para llegar a resultados si no se quedan en hipótesis, y diferentes los unos de los otros. Se ajustan bien a los principios.

4. Aplicación del modelo. Por falta de estadísticas suele ser poco precisa.

En general, estos modelos aportan siempre algo.

\section{Enfoques acturiles}

Citamos primero los que mejor conocemos.

Así:

Italia: Sr. Carpena, Sra. Manuzio. Participan en el Cigre (Asociación europea para material eléctrico), dando reglas semiprobabilísticas para líneas aéreas, teniendo en cuenta los factores anteriores. Estudiaron el viento y la nieve, y la consecuencia de daños. En general, tomaron como referencia la probabilidad de ruina anual integrando una ley de cargas y de resistencias.

Participan en el C.E.C.M. en la Comisión 1 (Carpena) y en el Grupo de Trabajo Ponderación (Director: Sr. Carpena; Vocal: Sra. Manuzio).

Holanda: El Sr. Loof, miembro del Grupo de Trabajo Ponderación de la Comisión 1 (Normas) del C.E.C.M., presentó un artículo del Laboratorio del Departamento de Ingeniería Civil de la Universidad Técnica de Delft, Holanda, de autor Kuipers. Se basa en la consideración del índice:

$$
f=(S-G-P) / \text { raíz de }\left(S_{s}^{2}+S_{g}^{2}+S_{p}^{2}\right),
$$

donde las leyes estadísticas de resistencias, cargas permanentes y vivas tienen medias $S, G, P$, varianzas $S_{s}, S_{g}, S_{p}$. Este índice es intuitivamente bueno, aunque si se usan leyes de valores extremos para cargas vivas, la influencia de las dispersiones de $S$ está infravalorada. Estudia influencias del valor de $f$ para va- 
rios materiales, y dispersiones, así como las relaciones entre $S$ y $P$ que dan mismo $f$, y por tanto seguridades comparables. Puede servir para dar forma a los coeficientes de seguridad.

En Estados Unidos hay varios focos de desarrollo del tema.

M. Cornell tiene publicados en ASCE (febrero 1967) monografía del MIT y papel interno del ASCE Task Committee on Structural Safety, varias consideraciones encaminadas a la práctica de la construcción, por ello con fuerte sentido a la vez ejecutivo y de realidad. Indica:

- Lo imperfecto del conocimiento de las leyes reales y de los cálculos estadísticos, que hace similares varias leyes de probabilidad, por no discriminar. Por ello usa leyes log-normales.

- Uso de cálculo plástico.

- En publicación ASCE (febrero 1967), una base estadística rigurosa en su teoría.

- En publicación interna del ASCE, Task Committee on Structural Safety, métodos prácticos. De modo similar el autor de este artículo usa (implícitamente) leyes log-normales. De modo parecido Torroja-Páez, considera productos $x$ y $z$.

$x=$ resistencia construyendo con cuidado/sin cuidado.

$y=$ resistencia cálculo/resistencia real material.

$z=$ resultado exacto/resultado con hipótesis empleados.

El producto sale log-normal y la dispersión es raíz de la suma de los cuadrados de las dispersiones. $V R / L=$ raíz de suma de cuadrados de todas las dispersiones.

- Indica ley $C=$ coeficiente entre medias $=\exp (A \times V R / L)$; donde $A$ varía con tablas dependiendo de la duración, consecuencias de la ruina, número de modos de posible fallo. Es un resultado por un método simplificado mucho por el uso de leyes log-normales.

- Aconseja varios niveles en las normas. Uno usual, otro con uso de fórmulas derivadas de leyes lognormales, otro con mejores estudios.

- Estudia la definición de «elementos». Deben ser claramente independientes. De nada sirve cortar una viga en trozos y sumar las probabilidades de ruina de cada trozo.

- Considera leyes de valores extremos para esfuerzos sísmicos como nosotros, incluso, para máximos anuales. Hace aplicaciones numéricas con datos U.S.A.

- Estudia relación entre probabilidad de ruina y óptimo coste de ruina. Para elemento y cargas dados, probabilidad y coste son inversamente proporcionales en primera aproximación.

Freudenthal en numerosos artículos en ASCE da desarrollos matemáticos. En particular da influencia del hiperestatismo que reduce riesgos para pocas aplicaciones de cargas. Turkstra y los señores Ang y Amin también tienen teorías matemáticas para el cálculo con otras consideraciones.

M. Thom (ASCE, marzo 56) tiene estudios completos y mapas sobre el viento en U.S.A. Usa leyes de valores extremos tipo II (nosotros por comodidad sólo usamos del tipo I).

Octavio A.-Rascón Chávez (México, ASCE, junio 67) ha hecho una brillante aportación sobre la fatiga, encaminada a la industria aeronáutica. Recordemos que la resistencia varía con la frecuencia de las cargas, por lo que se introduce una variable (frecuencia) más.

Estos autores citados son todos de primera línea. Omitimos muchos artículos y trabajos sobre el tema. En particular primeras figuras en las técnicas constructivas van a dar comunicaciones en un Simposio en Londres, septiembre 1969 (sobre nociones de seguridad en las estructuras y métodos de elaboración de proyectos).

Hay muy numerosos artículos de divulgación sobre la materia, y bastantes publicaciones sobre puntos limitados, en particular en países hispanoamericanos.

Después de haber redactado este artículo, tuvimos noticia de la Conferencia pronunciada por el Sr. Batanero en la apertura del curso académico 68-69, sobre el estado artual de las ideas sobre la seguridad de las estructuras; es la mejor introducción al tema que conocemos. 


\section{V. Considercaiones personules}

El tema está filosóficamente bien establecido. Los desarrollos matemáticos son insuficientes. La experimentación es escasa. La aceptación de la idea de «determinación probabilística» es buena en los medios internacionales.

Por creer que era lo más indicado, hemos seguido el camino siguiente:

- Aceptar las hipótesis simplificativas teóricas usuales, pero introduciendo el interés del dinero, que mide la preferencia de tenerlo ahora a tenerlo después, inspirados en los métodos de evaluación de inversiones. Usar la vida de la estructura. La influencia del primer factor es moderada, del segundo algo mayor.

- Usar leyes logarítmicas normales en vez de normales para las influencias que no dependen del tiempo. Como la diferencia es pequeña para dispersiones pequeñas, creemos está justificado. Recordamos que si una variable tiene su $\log N(A, S A)$, su media es próxima a exp $(A)$ su dispersión (varian$\mathrm{za} /$ media) a $S A$

En particular usamos productos de factores $x \cdot y \cdot z \cdot t \cdot u$, siguiendo a Torroja-Páez, para los que las leyes log-normales son más útiles.

- En cuanto que las cargas no son permanentes creemos que hay que usar leyes de valores extremos, $\mathrm{y}$ usamos las de tipo I. Hay casos en los que convienen las de tipo II $\mathrm{u}$ otras, pero para construir algo con principio y fin prescindimos de ellos.

Las leyes de valores extremos muy usadas por portuarios, tienen poca dispersión en los casos prácticos, y se desplazan a la derecha con el tiempo. En particular las de tipo I de función de distribución:

$$
\exp [-\exp (L(S-M)+\ln N)] \text {. }
$$

(De moda $M$, varianza $1,28 / L$, en tiempo $N$ ).

Para determinar la ley hay que fijar dos parámetros (con un valor del tiempo $N$ en el cual se aplican cargas). En otros serán durante la vida de la estructura supuesta aleatoria.

- Los parámetros naturales son la moda $Q$ y la desviación típica $Q$. $S Q$ de las leyes de valores extremos (lo que es definición de $S Q$ ). Para una ley de logaritmo normal $N(\ln R, S R$ ) se tomará como valor central $R$, como dispersión $S R$.

Para leyes de valores extremos hay que dar también el tiempo $N$ de aplicación de cargas. Hicimos nuestros cálculos con 10.000 días (27 años y pico) por parecernos cómodo. Se pueden dividir todos los intervalos de tiempo por un mismo número.

- Damos un modelo matemático para superposición de cargas independientes cuyos máximos no superponen, y ábacos (pendientes de reajustes).

- Damos tablas de valores óptimos de coeficientes de seguridad en función del coste de la estructura.

Los razonamientos obligan a distinguir, so pena de absurdos, incertidumbres de conocimiento y aleatoriedades de la naturaleza (cf., el incierto y aleatorio de Ferry Borges).

Los cálculos, bastante laboriosos, fueron hechos en el computador del Instituto Eduardo Torroja. Todavía hemos de hacer bastantes.

Hemos enviado por partes los trabajos al C.E.C.M. como papeles de uso interno; les escribimos tal vez con demasiada precipitación. Estamos revisándolos para publicarlos.

Recordemos que en toda teoría sobre coeficientes pueden, pero no suelen, tenerse en cuenta diversas reservas de resistencias, por lo que se obtienen unos coeficientes que no las tienen en cuenta.

Los coeficientes prácticos están acercándose a los teóricos que son menores, sobre todo para asociaciones de cargas.

Como hay muchas normas elaborándose, más que dar óptimos económicos, las teorías probabilistas pueden dar luz sobre superposición de cargas, y sobre todo unificar lenguajes y métodos en diversos países y para diversas técnicas. 


\title{
rósumb́ Summany
}

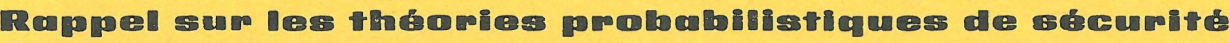

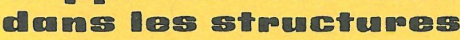

J. M. Antón Corrales, ingénieur des Ponts et Chaussées

Cet article rappelle brièvement à titre d'information, les principes, l'historique et le développement des théories probabilistiques pour déterminer les coefficients de sécurité. Il rappelle que l'on procède d'abord à la conception, puis au calcul et cite les principes déjà universels pour leur simplicité et évidence. II énonce brièvement quelques noms qui ont contribué à cette théorie, à laquelle l'auteur a travaillé à l'Institut Eduardo Torroja pour la C.E.C.M. (Convention Européenne de la Construction Métallique) sous la direction de J. Batanero.

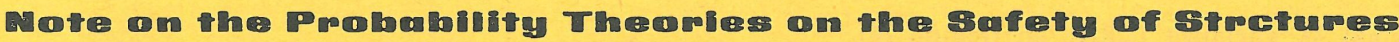

J. M. Antón Corrales, civil engineer

This informative article mentions briefly the principles, history and development of the probability theories available to determine the factors of safety. It reminds the reader that first a design is conceived and then the calculations follow, and it also mentions the universilly known principles which are as simple as they are evident. A brief mention is made of some of the names who contributed to the theory. The author has worked in this field at the Instituto Eduardo Torroja on behalf of the C.E.C.M. (European Convention of Metallic Construction), under the direction of J. Batanero.

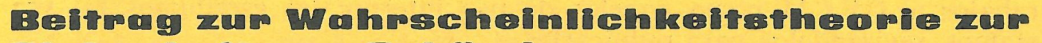

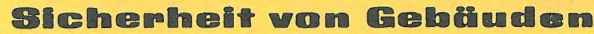

J. M. Antón Corrales, Bauingenieur

Der Artikel ruft kurz die Prinzipien, die Geschichte und die Entwicklung der Wahrscheinlichkeitstheorie von Sicherheitskoeffizienten ins Gedächtnis. Er erinnert daran, dass zunächst entworfen und dann berechnet wird und erwähnt die aufgrund ihrer Einfachheit und Klarheit bereits universellen Prinzipien. Er macht kurz auf einige Namen aufmerksam, die zu dieser Theorie beigetragen haben. Der Autor arbeitete hieran im Instituto Eduardo Torroja für die C.E.C.M. Convention Européenne de la Construction Métalique) unter der Aufsicht von J. Batanero. 